\title{
ArcheoSciences
}

Revue d'archéométrie

\section{Les objets en plomb découverts sur le site portuaire médiéval de Taillebourg - port d'Envaux}

typologie, fonction et origine

Lead artefacts found in Taillebourg - Port d'Envaux harbourg: Typology, use and origin

Florian Téreygeol, E. Foy, J.-F. Mariotti et A. Dumont

\section{OpenEdition}

\section{Journals}

\section{Édition électronique}

URL : https://journals.openedition.org/archeosciences/2818

DOI : $10.4000 /$ archeosciences. 2818

ISSN : 2104-3728

Éditeur

Presses universitaires de Rennes

Édition imprimée

Date de publication : 10 avril 2010

Pagination : 253-267

ISBN : 978-2-7535-1407-2

ISSN : 1960-1360

\section{Référence électronique}

Florian Téreygeol, E. Foy, J.-F. Mariotti et A. Dumont, « Les objets en plomb découverts sur le site portuaire médiéval de Taillebourg - port d'Envaux », ArcheoSciences [En ligne], 34 | 2010, mis en ligne le 11 avril 2013, consulté le 28 janvier 2022. URL : http://journals.openedition.org/archeosciences/ 2818 ; DOI : https://doi.org/10.4000/archeosciences.2818 


\title{
Les objets en plomb découverts sur le site portuaire médiéval de Taillebourg - port d'Envaux : typologie, fonction et origine
}

\author{
Lead artefacts found in Taillebourg - Port d'Envaux harbourg: \\ Typology, use and origin
}

F. Téreygeol ${ }^{*}$, E. Foy ${ }^{* *}$, J.-F. Mariotti ${ }^{* * *}$ et A. Dumont ${ }^{* * * *}$

\begin{abstract}
Résumé : Des prospections subaquatiques menées dans la Charente, sur le site de Taillebourg - Port d'Envaux (17), ont révélé la présence de nombreux témoins d'activités liés au fleuve Charente, datés principalement des périodes mérovingienne et carolingienne. Parmi l'abondant mobilier découvert figure un lot d'objets en plomb.

Ces plombs ont été trouvés dans un contexte de pratiques halieutiques. Les premières interprétations les définissent comme des lests d'engins de pêche. La découverte d'un tel corpus est déjà en soi un fait intéressant. En effet, la facilité de recyclage de ce matériau de plaide pas pour sa conservation. L'étude de ce matériel a porté à la fois sur la définition de leur typologie et une première interprétation de leur fonction. En plus des méthodes de classement habituellement utilisées, une approche expérimentale a été menée pour mieux appréhender les techniques de mise en forme utilisées. Des analyses de surface par fluorescence X ont pu être également réalisées. Enfin, le contexte chronologique plaçant cette collection au haut Moyen Âge et la découverte de ce matériel dans la zone d'influence de l'atelier monétaire de Melle nous ont conduit à poser la question de l'origine du plomb métal. Pour répondre à cette interrogation, une série d'analyses isotopique du plomb a été réalisée à partir de la série des plombs naviformes.
\end{abstract}

\begin{abstract}
Subaquaeous surveys carried out in the Charente, on the site Taillebourg - Port Envaux (dept. Charente-Maritime, France) revealed the presence of many evidences in activities related to river Charente, dating mainly from Merovingian and Carolingian periods. A lot of lead objects is found among the abundant furniture.

These leads were found in a context of fishing practices. The first interpretations defined as fishing gear. The discovery of such a corpus is itself an interesting fact. Indeed, the tendency of recycling this material does not plead for its preservation. The study of these sinkers has focused both on the definition of their type and initial interpretation of their function. In addition to classified methods usually used, an experimental approach was undertaken to better understand the techniques of production. Surface analysis by X-ray fluorescence were also performed. Finally, the historical context, placing the collection in the High Middle Ages and the discovery of this material in the zone of influence of the mint of Melle led us to the question of the origin of lead metal. To answer this question, a series of lead isotope analysis was carried out from the series of boat-form leads.
\end{abstract}

Mots clé : plomb de pêche, lest, Taillebourg, Melle, haut Moyen Âge, isotopie du plomb.

Key words: sinker, ballast, Taillebourg, Melle, early medieval time, lead isotopic analysis.

* UMR 5060 IRAMAT-LMC, Belfort et UMR 3299 SIS2M-LAPA, CEA - Saclay.

** UMR 3299 SIS2M-LAPA, CEA - Saclay.

*** SRA de Poitou-Charentes, Poitiers et UMR 5594 ARTeHIS - Anthropisation de l'environnement, Dijon.

**** DRASSM, Annecy et UMR 5594 ARTeHIS - Anthropisation de l'environnement, Dijon. 


\section{INTRODUCTION}

Bien que sûrement un des métaux les plus communément utilisés avec le fer, le plomb a paradoxalement laissé peu de traces tangibles dans les corpus archéologiques. La grande facilité qu'il y a à le refondre explique pour partie cet état de fait. Le recyclage n'étant pas une invention récente et le plomb fondant dès $327^{\circ} \mathrm{C}$, les objets usagés ou obsolètes pouvaient très facilement trouver le chemin du creuset pour une nouvelle vie. D'autre part, il faut bien reconnaitre que ce métal gris et mou n'a guère soulevé l'intérêt des archéologues. Tant et si bien que pour obtenir quelques éléments de comparaison, il est nécessaire de se tourner vers le monde Antique (Baron et Cochet, 2003; Cochet, 2000). Enfin, à ce jour, il n'a été retrouvé aucun atelier de plombier datant du Moyen Âge. Le fait est paradoxal puisque ce métal joue néanmoins un rôle important dans l'économie médiévale. Il sert directement à fabriquer de petits objets de la vie courante. Il entre dans la composition de l'alliage nécessaire à la fabrication de la poterie d'étain. Il est largement employé dans les travaux d'architecture (scellement, plomb de vitrail, couverture) tout comme dans les travaux urbains.

Cette absence d'un corpus étoffé justifie pleinement que l'on s'intéresse au lot d'objets mis au jour sur le site fluvial de Taillebourg - Port d'Envaux. Sa localisation en milieu immergé (à $8 \mathrm{~m}$ de profondeur), liée à des pertes accidentelles d'engins de pêche, a rendu toute récupération impossible et explique par là même sa richesse.

Il s'agit dans un premier temps de dresser un catalogue portant aussi bien sur la fonction ${ }^{1}$ que sur les techniques de mise en forme. La définition de la nature du métal employé est également poursuivie comme celle de l'origine du plomb. À terme, l'objectif est d'entamer la création d'un corpus d'objets en plomb pour la période médiévale. L'exemple charentais, souhaitons-le, devrait être enrichi ultérieurement par des plombs issus de cette même fouille mais également d'opérations archéologiques d'horizons variés.

\section{CONTEXTE DE DÉCOUVERTE : UNE ZONE PORTUAIRE DU HAUT MOYEN ÂGE}

Le château médiéval de Taillebourg, cité depuis 1007 dans les textes, dominait la Charente et permettait le contrôle des activités liées au fleuve (fig. 1). Construit sur un éperon rocheux, il contrôlait également le premier pont ren-

1. L'étude bibliographique n'a permis de localiser qu'un seul exemple de poids de pêche en France (Gruat, 2003) : lests en plomb, période médiévale sans précision, masses de 89 et $25 \mathrm{~g}$. Des exemples anglo-saxons existent également (Wallace P. F., 1998). contré lorsque l'on venait des côtes de l'Océan Atlantique. Carrefour de voies fluviales et terrestres, le secteur de Taillebourg est donc, durant toute la période médiévale un lieu stratégique. La zone concernée par la prospection subaquatique se trouve à $1 \mathrm{~km}$ en amont du bourg et du château. Elle a livré un nombre important d'embarcations (pirogues monoxyles et épaves assemblées), des structures (un aménagement de berge et une digue datés de la fin du $\mathrm{IX}^{\mathrm{e}}$ et du début du $\mathrm{X}^{\mathrm{e}}$ siècle), ainsi qu'un abondant mobilier (céramiques, outils, armes, lests en pierre et en plomb, etc.). Parmi ces objets, on compte plusieurs éléments qui trouvent des comparaisons typologiques dans l'Europe du Nord (anneau, haches) et qui confirment que les Vikings, au cours de leurs nombreux raids en Aquitaine, ont emprunté la Charente. Les textes mentionnent en effet la prise de la ville de Saintes en 845 (Debord, 1984).

Cette portion du fleuve a servi, pendant le haut Moyen Âge, de zone portuaire où les activités artisanales qui s'exerçaient sur les berges, le commerce, et la pratique de la pêche devaient tenir un rôle important. Taillebourg est situé à $40 \mathrm{~km}$ de l'Océan Atlantique mais l'influence de la marée, avant la construction des barrages, y était importante. Elle permettait la remontée des bateaux de mer ainsi que la possibilité de capturer des espèces de poissons variées (migrateurs notamment).

Les vestiges sont préservés aux abords de trois hauts-fonds entre lesquels se répartissent, par groupes distincts, les lests en plomb. Ces groupes correspondent vraisemblablement à des types de filets différents qui ont été perdus dans le fleuve, suite à des crues ou accrochés à des bois présents au fond (fig. 2).

\section{Catalogue}

Le catalogue qui suit regroupe l'ensemble des pièces en plomb retrouvées lors des opérations archéologiques subaquatique menées dans le fleuve Charente entre 2003 et 2007 (fig. 3). Il s'agit d'un lot de 85 objets répartis entre 10 familles. Cette grande variété du corpus ne permet pas de travailler avec une réelle approche statistique. Seules trois familles, représentant plus de $70 \%$ du corpus, peuvent conduire à une première réflexion sur les formes, les tailles et les masses. Pour les autres objets, des constatations isolées peuvent être produites dans l'attente d'un étoffement du nombre d'objets. Il est évident que la fonction principale de ces objets est à mettre en rapport avec les activités halieutiques pratiquées dans le fleuve. 


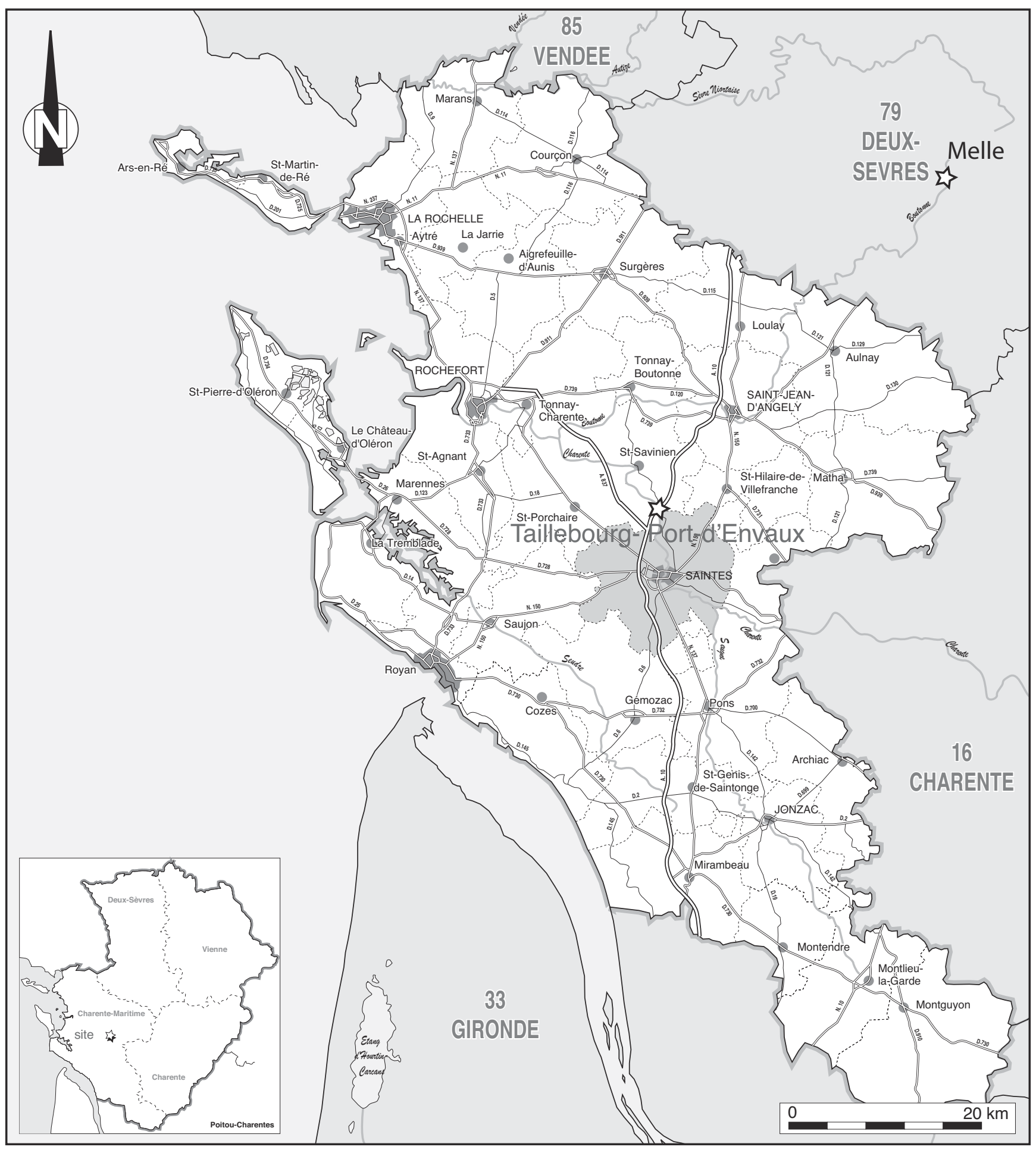

Figure 1 : Carte de localisation.

Figure 1: Map location. 


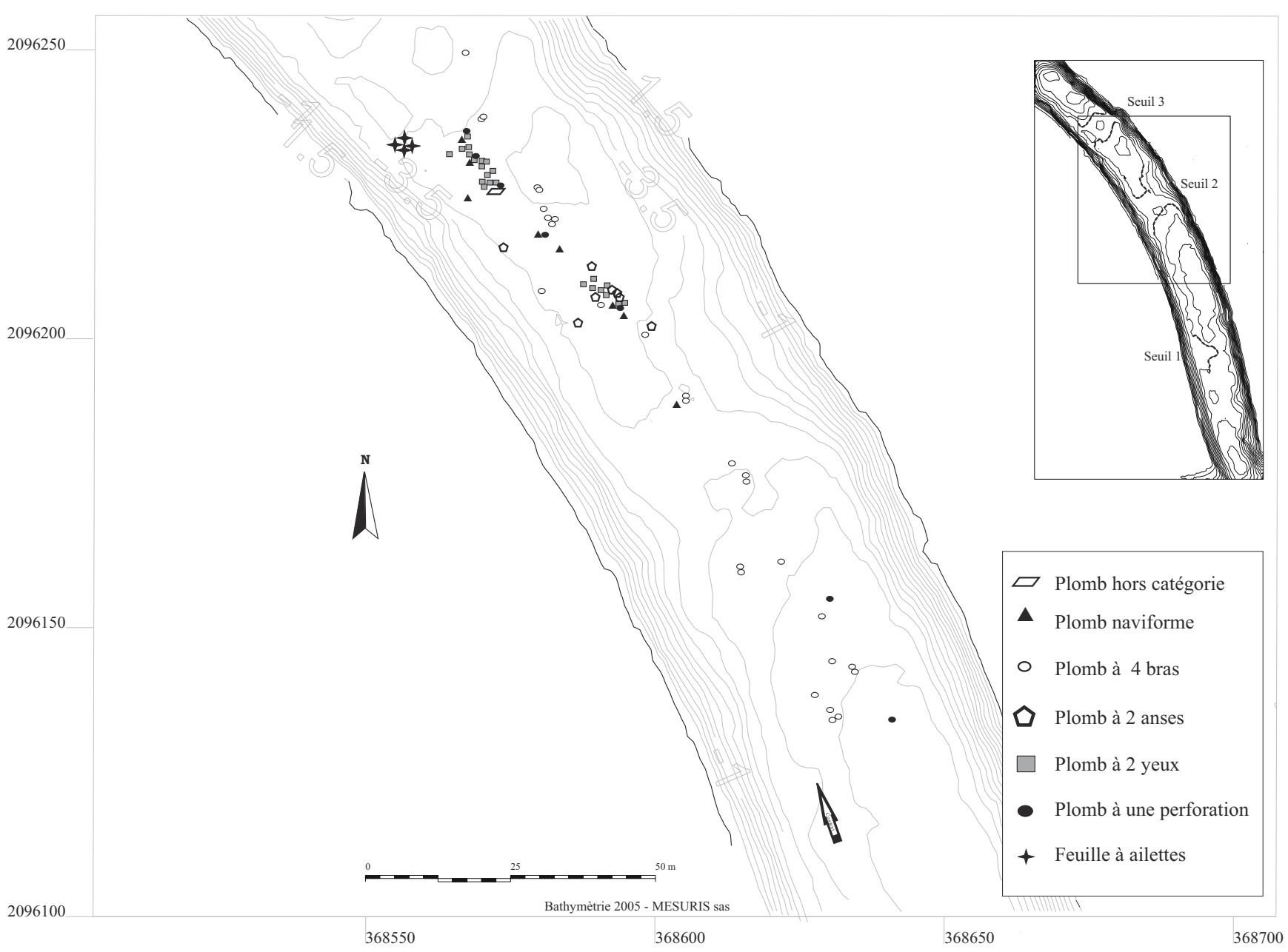

Figure 2 : Carte du lieu de découverte.

Figure 2: Discovery place.

\section{Plombs à 4 bras}

Il s'agit de barres de plomb fendues aux deux extrémités pour former deux bras devant être visiblement repliés en force sur l'objet à lester (fig. 4). Ces bras forment ce que nous appelons des "yeux ». Ces pièces, au nombre de 25 , représentent la plus grande famille du corpus. Elles ont, en moyenne, une longueur de $94 \mathrm{~mm}$ pour une largeur de $13 \mathrm{~mm}$ et une épaisseur de 7,6 mm. Leur masse d'une moyenne est de $84 \mathrm{~g}$. On note une légère tendance à la standardisation pour la longueur de ces barres. En revanche, ni la largeur, ni l'épaisseur ne montrent une volonté nette de reproduire le même objet. Il n'y a donc pas eu usage d'un même moule pour le corpus existant. Si l'objet varie dans ces dimensions et dans ses masses, il semble bien que le diamètre des yeux indique l'usage d'un même type de corde ayant entre 4 et $6 \mathrm{~mm}$ de section. Ces objets nécessitent de couler une barre ayant la morphologie et la masse voulue par rapport à l'objet final. L'usage de la coulée en sable permet d'être très facilement reproductible. Une fois la barre obtenue, les bras sont réalisés par découpe. Cette dernière est faite avec un taillant en percussion posée sur le plat de l'objet. Il s'en suit une reprise de découpe verticale dont la trace est très nettement visible sur les exemplaires archéologiques.

\section{Plombs à 2 anses}

Les plombs à deux anses sont au nombre de 16 (fig. 4). Il s'agit de masses ou de plaques comportant, dans la majeure partie des cas, deux excroissances dans lesquelles sont inscrits les yeux. Leur longueur moyenne est de $110 \mathrm{~mm}$ pour une largeur de 63,5 $\mathrm{mm}$ et une épaisseur de 9,7 $\mathrm{mm}$. La masse est 
Plomb à 4 bras

\begin{tabular}{|c|c|c|c|c|c|c|c|c|c|c|c|c|}
\hline \multicolumn{8}{|c|}{ Plomb à 4 bras } & \multicolumn{5}{|c|}{ dimensions des yeux $\mathrm{mm}$} \\
\hline & $n^{\circ}$ d'inv. & Description & Indices de surface & L mm & I mm & ép. mm & $\begin{array}{l}\text { Masse } \\
(\mathrm{g})\end{array}$ & L 1 & I1 & L 2 & 12 & IL 3 \\
\hline Tai 2003- & 156 & $\begin{array}{l}\text { Plomb à } 4 \text { bras formant deux yeux, } \\
\text { déformation en arc avec le point } \\
\text { d'inflexion au centre de l'objet. } \\
\text { Possibilité de coulée sur tranche }\end{array}$ & $\begin{array}{l}\text { Forme quandragulaire, irrégularités de coulée sur deux } \\
\text { bras formant l'un des yeux }\end{array}$ & 90 & 12,1 & 7 & 75,96 & 17 & 4 & 17 & 5,00 & \\
\hline Tai 2003- & 422 & Plomb à 4 bras ouverts (usure) & $\begin{array}{l}\text { Forme bipyramidale, usure prononcée des bras, traces } \\
\text { de découpe }\end{array}$ & 94 & 10,9 & 9,6 & 72,96 & ? & 5 & ? & 4,10 & \\
\hline Tai 2003- & 421 & Plomb à 4 bras ouverts (usure) & $\begin{array}{l}\text { Forme bipyramidale, usure prononcée des bras, traces } \\
\text { de découpe, surplus de matière sur les faces latérales } \\
\text { recouvrant les deux autres faces }\end{array}$ & 87,7 & 10,13 & 9,88 & 67,51 & ? & 6 & ? & 4,30 & \\
\hline Tai 2003- & 359 & Plomb à 4 bras ouverts (usure) & $\begin{array}{l}\text { Forme quadrangulaire, recouvrement d'un des bras par } \\
\text { un surplus de matière, mise en forme latérale } \\
\text { bidrirectionnelle, recourbement des bras }\end{array}$ & 98 & 11,8 & 9,5 & 114,87 & ? & 3 & ? & ? & \\
\hline Tai 2003- & 154 & Plomb à 4 bras formant deux yeux & $\begin{array}{l}\text { forme quadrangulaire, possibilité de soudure de } 2 \\
\text { barres }\end{array}$ & 105 & 14 & 7,6 & 110,6 & 21 & 5 & 23 & 4,70 & \\
\hline Tai 2003- & 386 & Plomb à 4 bras formant deux yeux & $\begin{array}{l}\text { Forme quandragulaire, irrégularité de coulée sur } 2 \text { bras } \\
\text { formant un œil, ouverture des yeux par découpe }\end{array}$ & 93,7 & 14 & 6 & 76,27 & 15 & 7 & 11 & 4,70 & \\
\hline Tai 2003- & 161 & Plomb à 4 bras formant deux yeux & Forme quandragulaire, ouverture des yeux par découpe & 106 & 11,45 & 7,26 & 95,46 & 12,7 & 5 & 16 & 5,00 & \\
\hline Tai 2003- & 420 & Plomb à 4 bras formant deux yeux & $\begin{array}{l}\text { Forme quandragulaire, ouverture des yeux par } \\
\text { découpe, recourbé par martelage et pincement, œil } \\
\text { ouvert }\end{array}$ & 92,8 & 10,5 & 8 & 66,4 & 9,4 & 5 & 9,2 & 5,60 & \\
\hline Tai 2003- & 302 & Plomb à 4 bras formant deux yeux & $\begin{array}{l}\text { Forme quandragulaire, apport de matière sur la face } \\
\text { interne, œil ouvert }\end{array}$ & 114 & 9,4 & 8,8 & 81,54 & 6,8 & $5 œ$ & 7,3 & 5,40 & \\
\hline Tai 2003- & 314 & Plomb à 4 bras totalement refermés & Forme quandragulaire, forte usure & 74,26 & 13,8 & 10,4 & 87,53 & ? & $?$ & $?$ & $?$ & \\
\hline Tai 2003- & 182 & Plomb à 4 bras formant deux yeux & $\begin{array}{l}\text { Forme quandragulaire, ouverture des yeux par découpe } \\
\text { et recourbés sur un bras }\end{array}$ & 92,95 & 11,55 & 6 & 60,55 & 15,9 & 4 & 16 & 4,00 & \\
\hline Tai 2003- & 155 & Plomb à 4 bras formant deux yeux & $\begin{array}{l}\text { Forme quandragulaire, ouverture des yeux par } \\
\text { découpe, yeux recourbés sur un bras }\end{array}$ & 95,14 & 14,1 & 7,5 & 100,11 & 14,2 & 4 & 14 & 4,30 & \\
\hline Tai 2003- & 387 & Plomb à 4 bras formant deux yeux & $\begin{array}{l}\text { Forme quandragulaire, ouverture des yeux par } \\
\text { découpe, un œil recourbé sur un bras, sur } 2 \text { pour l'autre }\end{array}$ & 106,05 & 13 & 6,5 & 94,73 & 12,5 & 6 & 11 & 5,70 & \\
\hline Tai 2004- & 112 & Plomb à 4 bras formant deux yeux & $\begin{array}{l}\text { Forme quandragulaire, ouverture des yeux par } \\
\text { découpe, objet non usagé, trace du taillant très nette }\end{array}$ & 93,72 & 10,84 & 8,59 & 78,29 & & 5 & & 2,30 & \\
\hline Tai 2004- & 109 & Plomb à 4 bras formant deux yeux & $\begin{array}{l}\text { Forme quandragulaire, ouverture des yeux par } \\
\text { découpe, trace du taillant très nette }\end{array}$ & 91,55 & 12,45 & 10,9 & 96,73 & & 5 & & 4,68 & \\
\hline Tai 2005 & $\begin{array}{l}\text { C121 } \\
\text { F133 }\end{array}$ & Plomb à 4 bras formant deux yeux & $\begin{array}{l}\text { Forme quandragulaire, ouverture des yeux par } \\
\text { découpe,tassement du corps }\end{array}$ & 100 & 16 & 5 & 85,27 & 20 & 7 & 22 & 8,00 & \\
\hline Tai 2005 & $\begin{array}{l}\text { C121 } \\
\text { F136 }\end{array}$ & Plomb à 4 bras formant deux yeux & $\begin{array}{l}\text { Forme quandragulaire, ouverture des yeux par } \\
\text { découpe, tassement du corps }\end{array}$ & 95 & 16 & 5,2 & 82,15 & 15 & 5 & 19 & 7,00 & \\
\hline Tai 2005 & $\begin{array}{c}\mathrm{C} 88 \\
\mathrm{~F} 114 \mathrm{~A}\end{array}$ & Plomb à 4 bras formant deux yeux & $\begin{array}{l}\text { Forme quandragulaire, ouverture des yeux par découpe } \\
\text { en deux temps. }\end{array}$ & 98 & 15 & 6,1 & 100,51 & 25 & 6 & 27 & 7,00 & \\
\hline Tai 2005 & $\begin{array}{c}\text { C88 } \\
\text { F114 B }\end{array}$ & Plomb à 4 bras formant deux yeux & $\begin{array}{l}\text { Forme quandragulaire, ouverture des yeux par } \\
\text { découpe, trace d'un raté de coulée }\end{array}$ & 110 & 15 & 5,6 & 98,55 & 27 & 7 & 30 & 5,00 & \\
\hline Tai 2005 & $\begin{array}{l}\text { C110 } \\
\text { F159 }\end{array}$ & Plomb à 4 bras formant deux yeux & $\begin{array}{l}\text { Forme quandragulaire, ouverture des yeux par } \\
\text { découpe, un œil excentré }\end{array}$ & 86 & 14 & 4 & 54,61 & 25 & 7 & 30 & 8,00 & \\
\hline Tai 2005 & $\begin{array}{l}\text { C121 } \\
\text { F110 } \\
\end{array}$ & Plomb à 4 bras formant deux yeux & $\begin{array}{l}\text { Forme quandragulaire, ouverture des yeux par } \\
\text { découpe, trace d'une reprise de découpe }\end{array}$ & 84 & 14 & 7,2 & 84,21 & 20 & 5 & 22 & 8,00 & \\
\hline Tai 2005 & $\begin{array}{l}\text { C121 } \\
\text { F134 }\end{array}$ & Plomb à 4 bras formant deux yeux & $\begin{array}{l}\text { Forme quandragulaire, ouverture des yeux par } \\
\text { découpe, trace d'une reprise de découpe }\end{array}$ & 74 & 18 & 4,5 & 60,34 & 22 & 7 & 18 & 7,00 & \\
\hline Tai 2005 & $\begin{array}{l}\text { C133 } \\
\text { F180 }\end{array}$ & Plomb à 4 bras formant deux yeux & $\begin{array}{l}\text { Forme quandragulaire incurvée, ouverture des yeux par } \\
\text { découpe, usure importante. }\end{array}$ & 87 & 10,5 & 12,4 & 94,77 & 10 & 5 & 9 & 6,00 & \\
\hline Tai2006 & 23 & Plomb à 4 bras formant deux yeux & $\begin{array}{l}\text { Forme quandragulaire, ouverture des yeux par } \\
\text { découpe, trace d'une reprise de découpe }\end{array}$ & 97 & 14,5 & 5,5 & 67,8 & & & & & \\
\hline Tai 2007 & 384 & Plomb à 4 bras formant deux yeux & $\begin{array}{l}\text { Forme quandragulaire incurvée, ouverture des yeux par } \\
\text { découpe, usure importante. }\end{array}$ & 85,5 & 12 & 12 & 102,9 & 10 & 5 & 9 & 6,00 & \\
\hline
\end{tabular}

Plomb à 2 anses

\begin{tabular}{|c|c|c|c|c|c|c|c|c|c|c|c|c|}
\hline \multicolumn{8}{|c|}{ Plomb à 2 anses } & \multicolumn{5}{|c|}{ dimensions des yeux $\mathrm{mm}$} \\
\hline & $n^{\circ}$ d'inv. & Description & Indices de surface & L mm & I mm & ép. $\mathrm{mm}$ & $\begin{array}{l}\text { Masse } \\
(\mathrm{g})\end{array}$ & L 1 & I1 & L 2 & 12 & IL 3 \\
\hline Tai 2003- & 454 & $\begin{array}{l}\text { Plomb à deux anses, Forme } \\
\text { trapézoïdale et départ en pyramide } \\
\text { permettant de restituer le sens de } \\
\text { coulée }\end{array}$ & $\begin{array}{l}2 \text { perforations circulaires dans les anses, débordement } \\
\text { lors de la coulée, oxyde de cuivre, empreintes en } \\
\text { croisillons (linge ?).les trous ont un diamètre minimum } \\
\text { de } 7.3 \text { et maxi de } 9.02 \text {, (sens de percement de la face } \\
\text { supérieure vers la face intérieure). }\end{array}$ & 69,9 & 62 & 10 & 388 & dia 7.3 & & & & \\
\hline Tai 2003- & 455 & $\begin{array}{l}\text { Plaque à deux perforations avec anses } \\
\text { mal définies }\end{array}$ & Retassure & 94 & 55 & 7,3 & 258 & dia 5.3 & & dia 5.3 & & \\
\hline Tai 2003- & 434 & $\begin{array}{l}\text { Plomb à deux anses, forme } \\
\text { quadrangulaire légèrement courbe }\end{array}$ & Allongement des perforations par usure & 132 & 69 & 11 & 774 & dia 7.36 & & dia 8 & & \\
\hline Tai 2004- & 16 & $\begin{array}{l}\text { Plomb quadrangulaire à deux anses, } 2 \\
\text { perforations }\end{array}$ & Ouverture "dentée" des yeux dans la partie supérieure & 107 & 71 & 9,9 & 520 & 7,5 & & 6 & & \\
\hline Tai 2004- & 48 & $\begin{array}{l}\text { Plomb quadrangulaire à deux anses, } 2 \\
\text { perforations }\end{array}$ & Retassure, pièce coulée en deux fois & 130 & 70 & 10 & 704 & 7,7 & & 8 & & \\
\hline Tai 2004- & 220 & Plaque à deux perforations avec anses & Replis volontaire des anses & 102 & 44 & 10 & 346 & 7,2 & & 8,4 & & \\
\hline Tai 2004- & 335 & $\begin{array}{l}\text { Plaque à deux perforations avec anses } \\
\text { mal définies }\end{array}$ & Traces de reprise de coulée, retassures & 113,5 & 67 & 7,8 & 320 & 7,3 & & 7,5 & & \\
\hline Tai 2004- & 369 & Plaque à deux perforations avec anses & & 114 & 70 & 7 & 395 & 7 & & 6,5 & & \\
\hline Tai 2004- & 172 & Plaque à deux perforations avec anses & & 120 & 64 & 14 & 630 & 6,6 & & 6,7 & & \\
\hline Tai 2004- & 159 & Plaque à deux perforations avec anses & Une anse mal formée, coulée dans le sol & 111 & 67 & 8,5 & 416 & 7,5 & & 7,7 & & \\
\hline Tai 2004- & 155 & Plaque à deux perforations avec anses & Anses mal formées & 113 & 63 & 8 & 294 & 7,5 & & 5 & & \\
\hline Tai 2005 & 292 & $\begin{array}{l}\text { Plomb à deux anses, forme } \\
\text { quadrangulaire }\end{array}$ & $\begin{array}{l}\text { Moulage irrégulier, traces de découpe pour ajustement, } \\
\text { un œil issu d'une réserve, l'autre suite à une perforation } \\
\text { par une pointe quadrangulaire }\end{array}$ & 125 & 70 & 8,4 & 565 & 8 & & 6 & & \\
\hline Tai 2005 & 264 & $\begin{array}{l}\text { Plomb à deux anses, forme } \\
\text { quadrangulaire légèrement courbe }\end{array}$ & une anse mal formée, coulée dans le sol & 112 & 41 & 5,5 & 360 & 6 & & 14 & 8 & \\
\hline Tai 2007 & 252 & Plaque à deux perforations avec anses & une perforation circulaire, une quandragulaire & 95 & 56 & 19 & 556,3 & & & & & \\
\hline Tai 2007 & 145 & Plaque à deux perforations avec anses & une perforation arrachée & 144 & 67,5 & 9 & 675,1 & & & & & \\
\hline Tai 2007 & 144 & Plaque à deux perforations avec anses & & 76 & 80 & 10,5 & 494,5 & & & & & \\
\hline
\end{tabular}


Plomb naviForme

\begin{tabular}{|c|c|c|c|c|c|c|c|c|c|c|c|c|}
\hline \multicolumn{8}{|c|}{ Plomb naviForme } & \multicolumn{5}{|c|}{ dimensions des yeux $\mathrm{mm}$} \\
\hline & $n^{\circ} d^{\prime}$ inv. & Description & Indices de surface & $\mathrm{L} \mathrm{mm}$ & I mm & ép. mm & $\begin{array}{l}\text { Masse } \\
\text { (g) }\end{array}$ & L 1 & 11 & L2 & 12 & IL 3 \\
\hline Tai 2002 & 131 & Plomb naviforme & & 124 & 97 & 13,4 & 975,9 & 36 & 33 & 27 & 29,3 & \\
\hline Tai 2003- & 160 & $\begin{array}{l}\text { Plomb à trois anses, forme } \\
\text { quadrangulaire }\end{array}$ & Défaut de coulée, perforations circulaires & 88,8 & 72 & 11 & 566 & dia 7.2 & & 7,6 & & 7,3 \\
\hline Tai 2004- & 275 & Plomb naviforme & $\begin{array}{l}\text { Forme circulaire à la base, les ouvertures ont été } \\
\text { formées lors de la coulée }\end{array}$ & 92 & 76 & 9 & 391 & 28 & 17 & 27 & 23 & \\
\hline Tai 2004- & 158 & $\begin{array}{l}\text { Plomb naviforme à trois anses } \\
\text { perforées }\end{array}$ & $\begin{array}{l}\text { Décor linéaire, la réserve circulaire est plus large que } \\
\text { les } 2 \text { autres qui ont un diamètre identique }\end{array}$ & 110 & 55,6 & 14,7 & 590 & 6,3 & & 8,2 & & 6,3 \\
\hline Tai 2004- & 178 & Plomb naviforme & traces de moule en bois (?) & 118 & 70 & 9,5 & 436 & 38 & 33 & 41 & 33 & \\
\hline Tai 2004- & 119 & Plomb naviforme & $\begin{array}{l}\text { Plomb replié, traces de martelage, ou de pinces, pièce } \\
\text { destinée à la refonte (?) }\end{array}$ & 128 & 56,5 & 20,4 & 918 & & & & & \\
\hline Tai 2005 & F 258 & Plomb naviforme & Défaut de coulée, surplus de métal, décor linéaire & 90 & 70 & $\frac{6}{2}$ & 265 & 12 & 10 & 20 & 17 & \\
\hline Tai 2005 & $\begin{array}{l}\mathrm{C} 135 \\
\mathrm{~F} 120\end{array}$ & $\begin{array}{l}\text { Plomb à trois anses, forme } \\
\text { quadrangulaire }\end{array}$ & $\begin{array}{l}\text { Trois ouvertures dont une formée lors de la coulée, les } \\
2 \text { autres formées par replis }\end{array}$ & 85 & 60 & 8,1 & 350 & 7 & & 7 & & 7 \\
\hline Tai 2005 & $\begin{array}{l}\mathrm{C} 122 \\
\mathrm{~F} 215\end{array}$ & Plomb naviforme & $\begin{array}{l}\text { Objet en cours de refonte, présence d'une erreur lors } \\
\text { de la fabrication du moule }\end{array}$ & 72 & 50 & 3 & 105,1 & & & & & \\
\hline Tai 2006 & 72 & Plaque avec 3 perforations & & 132 & 99 & 19 & 1089,6 & & & & & \\
\hline Tai 2007 & 55 & Plaque avec 3 perforations & & 91 & 63,5 & 14 & 454,2 & & & & & \\
\hline Tai 2007 & 183 & $\begin{array}{l}\text { Plomb à trois anses, forme } \\
\text { quadrangulaire }\end{array}$ & & 84 & 79 & 11 & 434,1 & & & & & \\
\hline Tai 2007 & 259 & $\begin{array}{l}\text { Plomb à trois anses, forme } \\
\text { quadrangulaire }\end{array}$ & & 84,3 & 79 & 11 & 396,9 & & & & & \\
\hline Tai 2007 & 366 & $\begin{array}{l}\text { Plomb à trois anses, forme } \\
\text { quadrangulaire }\end{array}$ & & 72,5 & 60 & 13 & 377,4 & & & & & \\
\hline Tai 2007 & 140 & $\begin{array}{l}\text { Plomb à trois anses, forme } \\
\text { quadrangulaire }\end{array}$ & & 90 & 67,5 & 12,5 & 444,3 & & & & & \\
\hline Tai 2007 & 203 & Plomb naviforme & & 104 & 60,5 & 9,5 & 333,7 & & & & & \\
\hline Tai 2007 & 188 & Plomb naviforme & mal fondu & 90 & 79,5 & 10 & 302,5 & & & & & \\
\hline Tai 2007 & $143 \mathrm{~b}$ & Plomb naviforme & & 104,5 & 64,5 & 4 & 528,7 & & & & & \\
\hline
\end{tabular}

\section{Plomb massif}

\begin{tabular}{|c|c|l|l|}
\hline & $n^{\circ}$ d'inv. & Description & Indices de surface \\
\hline Tai 2004- & 43 & $\begin{array}{l}\text { Plomb quadrangulaire à deux } \\
\text { perforations }\end{array}$ & $\begin{array}{l}\text { Mise en forme par martelage, empreintes circulaires } \\
\text { autour des yeux (avec métal repoussé) }\end{array}$ \\
\hline Tai 2004- & 315 & $\begin{array}{l}\text { Plomb quadrangulaire à deux } \\
\text { perforations }\end{array}$ & $\begin{array}{l}\text { Perforations faites de la face supérieure vers la face } \\
\text { inférieure }\end{array}$ \\
\hline
\end{tabular}

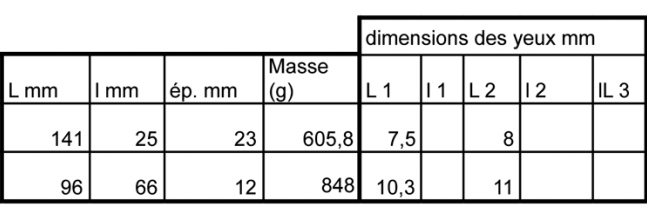

Barre

\begin{tabular}{|c|c|l|l}
\hline & $n^{\circ}$ d'inv. & Description & Indic quadrangulaire à deux \\
\hline Tai 2004- & 31 & $\begin{array}{l}\text { perforations disposées dans la } \\
\text { longueur }\end{array}$ & ysu \\
\hline
\end{tabular}

Indices de surface

Usure des passants, nombreux replis au niveau des yeux, pièce coulée dimensions des yeux $\mathrm{mm}$ \begin{tabular}{|l|l|l|l|l|l|l|l|l|}
\hline $\mathrm{mm}$ & $\mathrm{I} \mathrm{mm}$ & ép. $\mathrm{mm}$ & $\begin{array}{l}\text { Masse } \\
(\mathrm{g})\end{array}$ & $\mathrm{L} 1$ & $\mathrm{I} 1$ & $\mathrm{~L} 2$ & $\mathrm{I} 2$ & $\mathrm{IL} \mathrm{3}$ \\
\hline & & & & & & & & \\
92 & 16 & 16 & 192,64 & 5,4 & & 4,7 & & \\
\hline
\end{tabular}

\section{Plomb circulaire}

\begin{tabular}{|c|c|c|c|c|c|c|c|c|c|c|c|c|}
\hline \multicolumn{8}{|c|}{ Plomb circulaire } & \multicolumn{5}{|c|}{ dimensions des yeux mm } \\
\hline & $n^{\circ}$ d'inv. & Description & Indices de surface & $L \mathrm{~mm}$ & $\mathrm{Imm}$ & ép. $\mathrm{mm}$ & $\begin{array}{l}\text { Masse } \\
(\mathrm{g})\end{array}$ & L 1 & 11 & L 2 & 12 & IL 3 \\
\hline Tai 2004- & 307 & $\begin{array}{l}\text { Plomb circulaire percé d'un trou en } \\
\text { périphérie }\end{array}$ & Moule mal constitué, épaississement des bords & 82 & 62 & trion & 410 & 8,62 & & & & \\
\hline Tai 2004- & 179 & $\begin{array}{l}\text { Plomb circulaire percé d'un trou en son } \\
\text { milieu }\end{array}$ & percement du trou par les deux faces & 79 & 73 & 10,6 & 380 & 9,1 & & & & \\
\hline Tai 2005- & $\begin{array}{l}\text { C122 } \\
\text { B214 }\end{array}$ & Masse de plomb circulaire avec un œil & $\begin{array}{l}\text { Masse coulée à même le sol, œil fait par une réserve } \\
\text { lors de la coulée }\end{array}$ & 80 & 60 & 15 & 490 & 18 & & & & \\
\hline
\end{tabular}

Feuille à ailettes

\begin{tabular}{|l|c|l|l|}
\hline & $n^{\circ} d^{\prime}$ 'inv. & Description & In \\
\hline Tai 2004- & $262-1$ & Feuille à aillettes roulées & M \\
\hline Tai 2004- & $262-2$ & Feuille à aillettes roulées & M \\
\hline Tai 2004- & $262-3$ & Feuille à aillettes roulées & M \\
\hline Tai 2004- & $262-4$ & Feuille à aillettes roulées & des \\
\hline
\end{tabular}

ndices de surface

Martelage de la barre pour former les aillettes

Martelage de la barre pour former les aillettes

Martelage de la barre pour former les aillettes, soudure des ailettes (?)

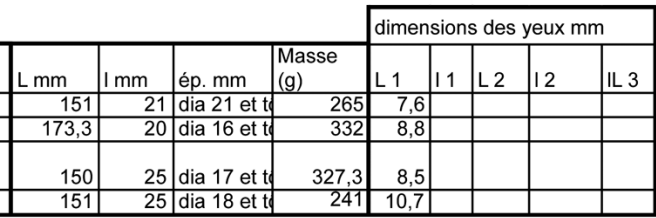

\section{Masse informe}

Masse informe
\begin{tabular}{|c|c|l|l|}
\hline & $n^{\circ}$ d'inv. & Description & Indices de surface \\
\hline Tai 2003- & 456 & $\begin{array}{l}\text { Masse de plomb avec une perforation } \\
\text { circulaire }\end{array}$ & $\begin{array}{l}\text { Replis du métal, masse coulée en moule avec une } \\
\text { quantitée insuffisante de matière }\end{array}$ \\
\hline Tai 2004- & 154 & $\begin{array}{l}\text { Masse de plomb avec une perforation } \\
\text { circulaire }\end{array}$ & Masse avec replis multiples \\
\hline Tai 2004- & 177 & Feuille de plomb, 2 perforations & $\begin{array}{l}\text { Feuille de plomb repliée sur elle-même avec un repli de } \\
\text { renfort sur un bord }\end{array}$ \\
\hline Tai 2004- & 353 & Plaque à deux perforations & Coulée sur bois (?) \\
\hline Tai 2007 & 190 & $\begin{array}{l}\text { Masse de plomb avec une perforation } \\
\text { circulaire }\end{array}$ & \\
\hline Tai 2007 & $268 a$ & Raté de coulée & \\
\hline
\end{tabular}

\begin{tabular}{|c|c|c|c|c|c|c|c|c|}
\hline & & & & dimen & sions & $s$ des & yeux & \\
\hline L mm & $\mathrm{Imm}$ & ép. mm & \begin{tabular}{|l} 
Masse \\
$(\mathrm{g})$
\end{tabular} & L 1 & 11 & $\mathrm{~L} 2$ & 12 & IL 3 \\
\hline 70 & 46 & 14 & 209,1 & 7,4 & & & & \\
\hline 121,4 & 37,8 & 16,5 & 275 & 8,8 & 6 & & & \\
\hline 130 & 68 & 8,16 & 351 & 5,24 & & 5,2 & & \\
\hline 89 & 59 & 7 & 343 & 10,8 & & 11 & & \\
\hline 80 & 54 & 9 & 183,4 & & & & & \\
\hline 42,5 & 45 & 4 & 28,8 & & & & & \\
\hline
\end{tabular}




\section{Coulure}

\begin{tabular}{|c|c|c|c|c|c|c|c|}
\hline & $n^{\circ}$ d'inv. & Description & Indices de surface & $\mathrm{L} \mathrm{mm}$ & $\mathrm{I} \mathrm{mm}$ & ép. $\mathrm{mm}$ & \begin{tabular}{|l|} 
Masse \\
$(\mathrm{g})$
\end{tabular} \\
\hline Tai 2004- & 61 & Coulure de plomb & & 47 & 24 & 9,9 & 24,95 \\
\hline Tai 2004- & C15 & Coulure de plomb & & 26 & 7 & 5 & 3,11 \\
\hline Tai 2004- & 188 & Coulure de plomb & & 44 & 28 & 10 & 21,59 \\
\hline Tai 2004- & 153 & Coulure de plomb & & 57 & 22 & 10 & 22,91 \\
\hline Tai 2004- & 334 & 2 coulures de plomb & $\begin{array}{l}\text { Fragments de charbon sur l'un avec un fragment de } \\
\text { scorie }\end{array}$ & & & & $\begin{array}{l}62.62 \text { et } \\
77.66\end{array}$ \\
\hline Tai 2004- & 351 & Lot de coulures de plomb & & & & & 15,03 \\
\hline Tai 2004- & 253 & Fragment de Plomb & Traces de découpe et de martelage de la pièce coulée & & & & 366 \\
\hline
\end{tabular}

\section{Divers}

\begin{tabular}{|c|c|c|c|c|c|c|c|}
\hline & $n^{\circ} d^{\prime}$ inv. & Description & Indices de surface & L mm & $\mathrm{Imm}$ & ép. mm & $\begin{array}{l}\text { Masse } \\
(\mathrm{g})\end{array}$ \\
\hline Tai 2003- & 430 & Bille percée & Perforation cylindrique $4.25 \mathrm{~mm}$ & dia 16 & & 11,95 & 16,78 \\
\hline Tai 2003- & 152 & Jeton circulaire & Traces de pinces sur le pourtour & dia 25 & & 3,34 & 15,56 \\
\hline Tai 2003- & 199 & Tôle roulée & Forme tubulaire non jointive & 84 & & 2,5 & 59,35 \\
\hline
\end{tabular}

Figure 3 : Liste des objets en plomb.

Figure 3: list of lead items.
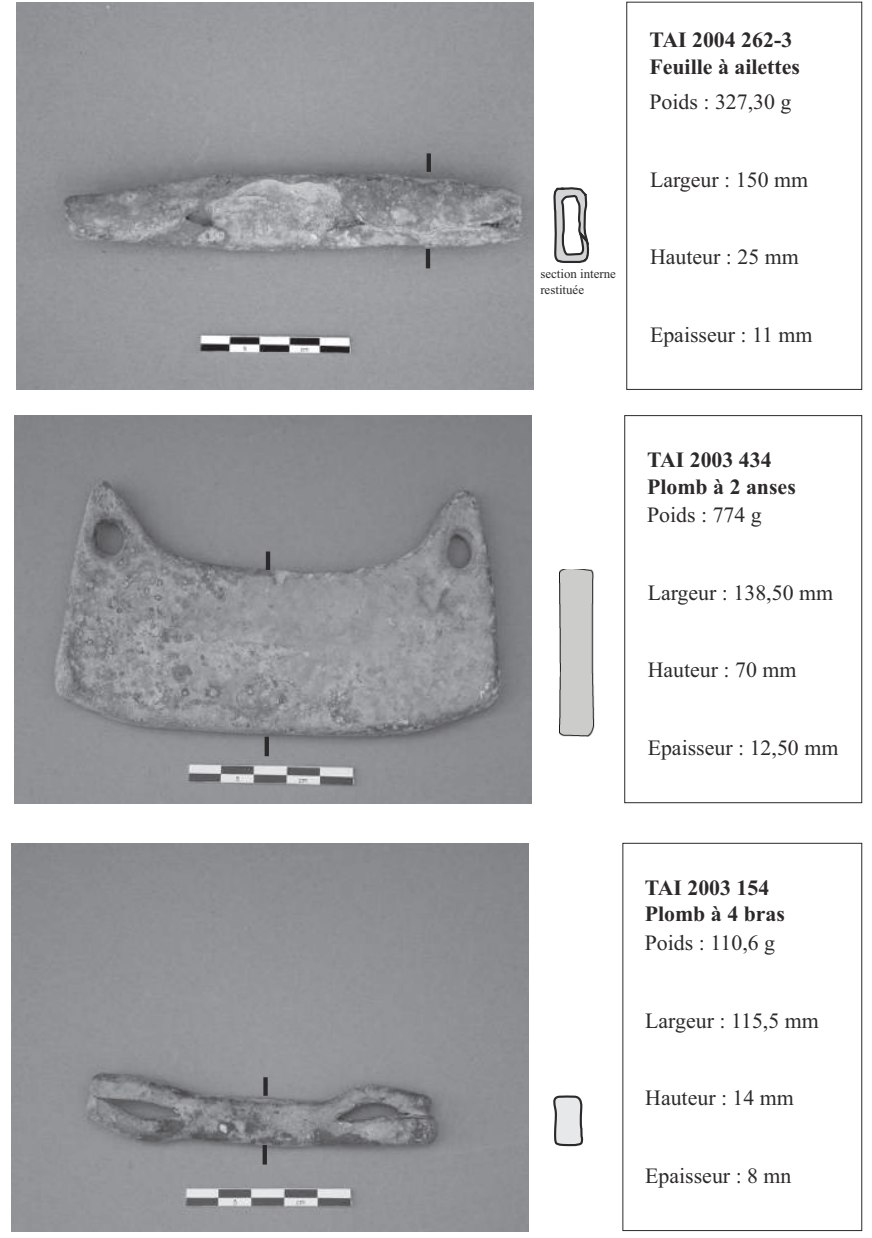
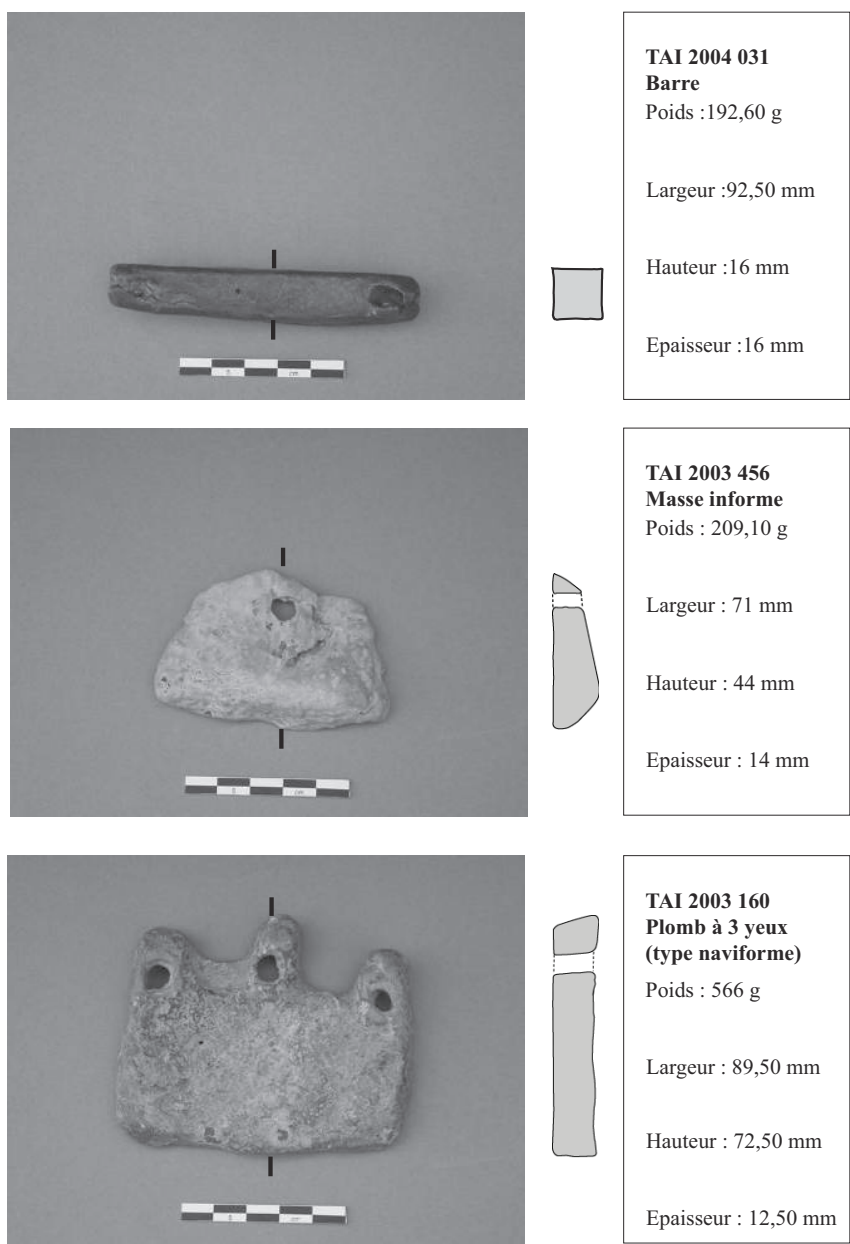

TAI 2003456

Masse informe

Poids : 209,10 g

Largeur : $71 \mathrm{~mm}$

Hauteur : $44 \mathrm{~mm}$

Epaisseur : 14 mm

TAI 2003160

Plomb à 3 yeux

(type naviforme)

Poids : $566 \mathrm{~g}$

Largeur : $89,50 \mathrm{~mm}$

Hauteur : 72,50 mm
Epaisseur : 12,50 mm

Figure 4 : Exemples des different plombs retrouvés dans la Charente. Figure 4: Examples of differents kinds of lead found in river Charente. 
très variable allant de 258 à $774 \mathrm{~g}$ sans qu'une valeur moyenne significative puisse être avancée montrant une absence de standardisation quant à la masse recherchée. Mais, comme dans le cas précédent, les yeux ont des diamètres homogènes : entre 6 et $8 \mathrm{~mm}$ pour $80 \%$ du corpus. Cette dimension supérieure à celle observée pour les plombs à 4 bras doit-elle être mise en relation avec la masse de l'objet et, donc, avec sa fonction de lest? L'étude des techniques de pêche devrait permettre d'apporter des éléments de réponse. Le seul problème technique de ces plombs de suspension repose sur la réalisation des yeux. Il ne s'agit pas de perforations qui auraient entraîné une déformation mécanique de la pièce tant au niveau des yeux (bourrelets de métal) que sur les bords de la plaque. L'usage de deux morceaux de bois permet à moindre coût de réaliser une réserve pour former ces ouvertures directement lors de la fonte. Une restitution expérimentale de la pièce TAI03-454 a permis par cette méthode de recréer une pièce très semblable, au détail prés que l'objet archéologique a connu un défaut de coulée (surplus de métal). Ce mode de réalisation est valable pour quasiment tous les yeux rencontrés dans ce corpus à l'exclusion de TAI 04-16 pour lequel la perforation a été faite à l'aide d'un foret, et pour les exemplaires TAI 04-43 et 335 dont les yeux ont été réalisés par perforation à l'aide d'une pointe.

\section{Feuilles à ailettes}

Les feuilles à ailettes sont au nombre de 4 avec une longueur moyenne de $156 \mathrm{~mm}$, une largeur de $22 \mathrm{~mm}$ et une épaisseur de $8 \mathrm{~mm}$ (fig. 4). La masse moyenne est de $291 \mathrm{~g}$ avec un diamètre des passants de $8,9 \mathrm{~mm}$. Il faut noter l'absence de soudures des ailettes deux à deux. On peut en conclure qu'il ne devait pas y avoir une tension trop forte exercée sur la pièce car les ailettes se seraient rapidement ré-ouvertes. Il s'agit visiblement de lester des fils ne devant pas être mis en forte charge. Les 4 pièces retrouvées l'ont été avec les ailettes correctement jointes. La perte ne s'est donc pas faite pendant l'usage de l'engin de pêche auquel elles étaient liées. Si l'objet paraît simple, il s'agit sûrement de celui nécessitant le plus d'étapes de mise en forme. Il faut travailler à partir d'une petite barre de plomb. Sa confection commence par la coulée de celle-ci à une épaisseur proche de l'objet achevé. En optant pour la mise en forme la plus simple, sa réalisation devait se faire dans un moule ouvert. Un rapide martelage réduit l'épaisseur du corps en chassant du métal vers les extrémités. Il s'ensuit un amincissement volontaire des extrémités qui produit une ailette de forme grossièrement circulaire. Suffisamment élargie, elle est peutêtre alors roulée sur elle-même de façon à créer la formes tubulaire des extrémités. Ici encore l'approche expérimentale a conduit à la reproduction d'une pièce similaire à celles observées dans ce corpus (fig. 5).

\section{Plombs naviformes}

Il s'agit sûrement des pièces les plus emblématiques de ce corpus (fig. 6). Elles évoquent la silhouette d'un bateau symétrique avec les proues et poupes verticales, le mât central et, pour celles qui possèdent des rainures horizontales, une coque assemblée à clin. Ces pièces naviformes ressemblent aux nombreuses figurations de bateaux que l'on rencontre dans le monde scandinave (Mariotti et al., 2005, fig. 12). Pour le moment, aucun objet comparable n'a été trouvé dans la littérature disponible. Cet état de fait est lié à la rareté des sites fluviaux du haut Moyen Âge fouillés en Europe. Les informations dont nous disposons actuellement ne permettent pas de savoir si une base viking a existé à Taillebourg ou si ces objets ont été fabriqués par une population qui a été en contact direct avec les Scandinaves qui, lorsqu'ils ne pillaient pas, s'adonnaient aux échanges et au commerce. $\mathrm{Si}$ ces plombs restent dans un contexte propre au fleuve Charente, une partie de cette famille pourrait avoir connu un usage de marquage des engins de pêches (grands filets probablement), afin que le propriétaire du filet (et de la capture) soit clairement identifié. Ces plombs naviformes n’ont pas été découverts regroupés en un seul point du site, mais répartis dans plusieurs secteurs du chenal, associés à des plombs d'autres types, ce qui pourrait conforter l'hypothèse de leur rôle de marqueur de filets.

Sur l'ensemble de ce lot, aucune corrélation n'apparaît entre les longueurs, largeurs, épaisseurs et masses des différentes pièces (longueur moy. 98 [ec 18], largeur moy. $70 \mathrm{~mm}$ [ec 13), épaisseur moy. $13 \mathrm{~mm}$ [ec 4,5], masse $497 \mathrm{~g}$ [ec 255]). En revanche, il est clair que les pièces TAI 02-131, 04-119, 05-215 et 05-258 sont faites sur le même modèle : la représentation figurative d'une embarcation. Il est même possible de proposer une filiation stylistique entre les pièces de ce corpus partant du groupe original dont l'exemplaire TAI 02-131 est le mieux conservé, et aboutissant d'une part au plomb type TAI04-175 (plomb figuratif) et d'autre part au plomb type TAI03-160 (plomb de suspension). Ces pièces coulées ont-elles pu être réalisées rapidement en série? La découverte de quatre exemplaires similaires le laisse croire. En partant de cette hypothèse, le moyen le plus simple pour y parvenir a été recherché. Une première expérience a été tentée avec du sable de fonderie moderne. Une fois le sable tassé dans son cadre, on sculpte en négatif le motif à produire. La relative simplicité du modèle rend l'opération aisée. Lors du démoulage de la première fonte, le négatif n'est pas réutilisable mais cette première épreuve peut être facilement employée comme positif 


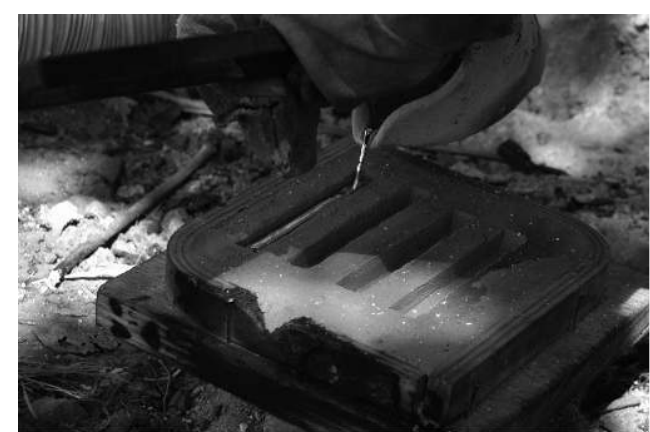

Coulée des barres

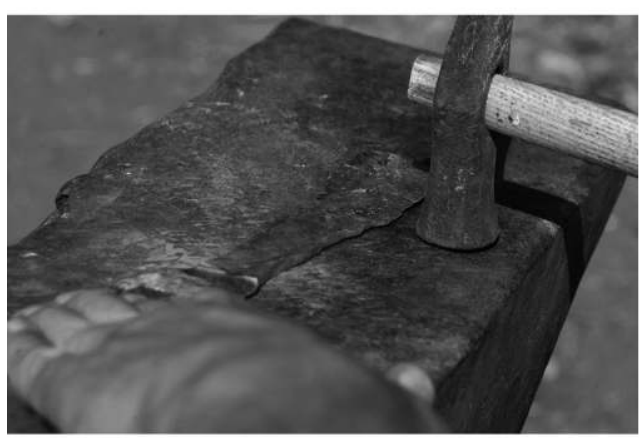

Martelage pour affiner les extrémités formant les ailettes

Ailettes à plat et mise en forme

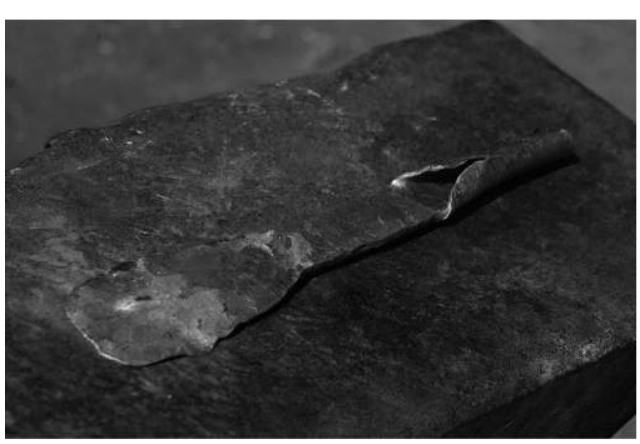

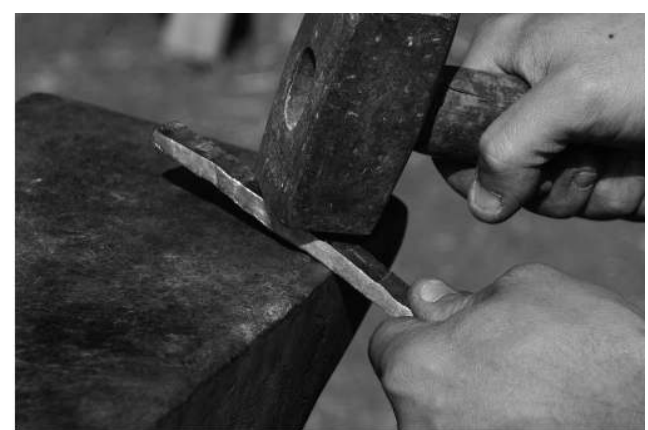

Martelage succint pour amener la barre à l'épaisseur voulue

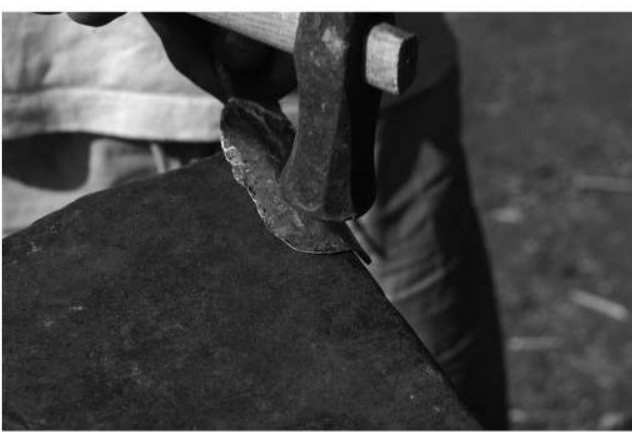

Repli de l'ailette

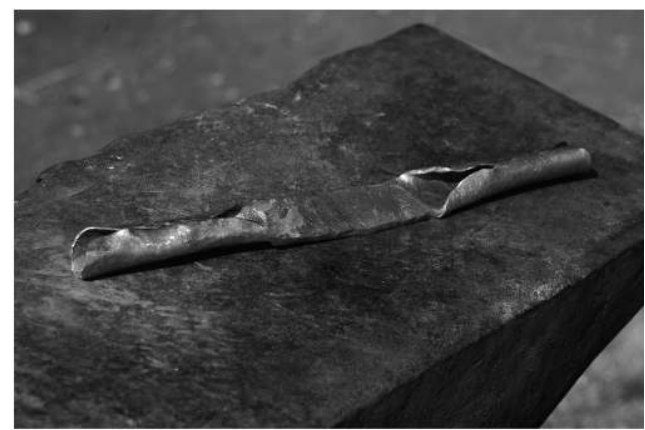

Plomb à ailettes
Figure 5 : Mise en forme expérimentale des plombs à ailettes. Figure 5: Experimental making up of wing-lead. tions typologiques observées. Bien que rares, des moules en pierre existent pour la réalisation d'objet en plomb (Lautier et Sandron, 2008; Arles et Téreygeol, 2009) tout comme nous en connaissons pour les moules en argile ${ }^{2}$. Les fontes dans ces types de matériau ne peuvent être exclues. Mais elles présentent des inconvénients en termes de rapidité d'exécution. De ces quatre voies de production possibles, nous privilégions la fonte au sable pour des raisons techniques et pratiques. La réalisation de pièces moulées comportant un décor en basrelief et/ou d'importantes réserves en rapport du volume mis

2. On citera par exemple : Murat L., 2007, Eglise Saint-Christophe, Commune de Montsaunès (31), rapport d'opération préventive, éd. Hadès, 41 p. 

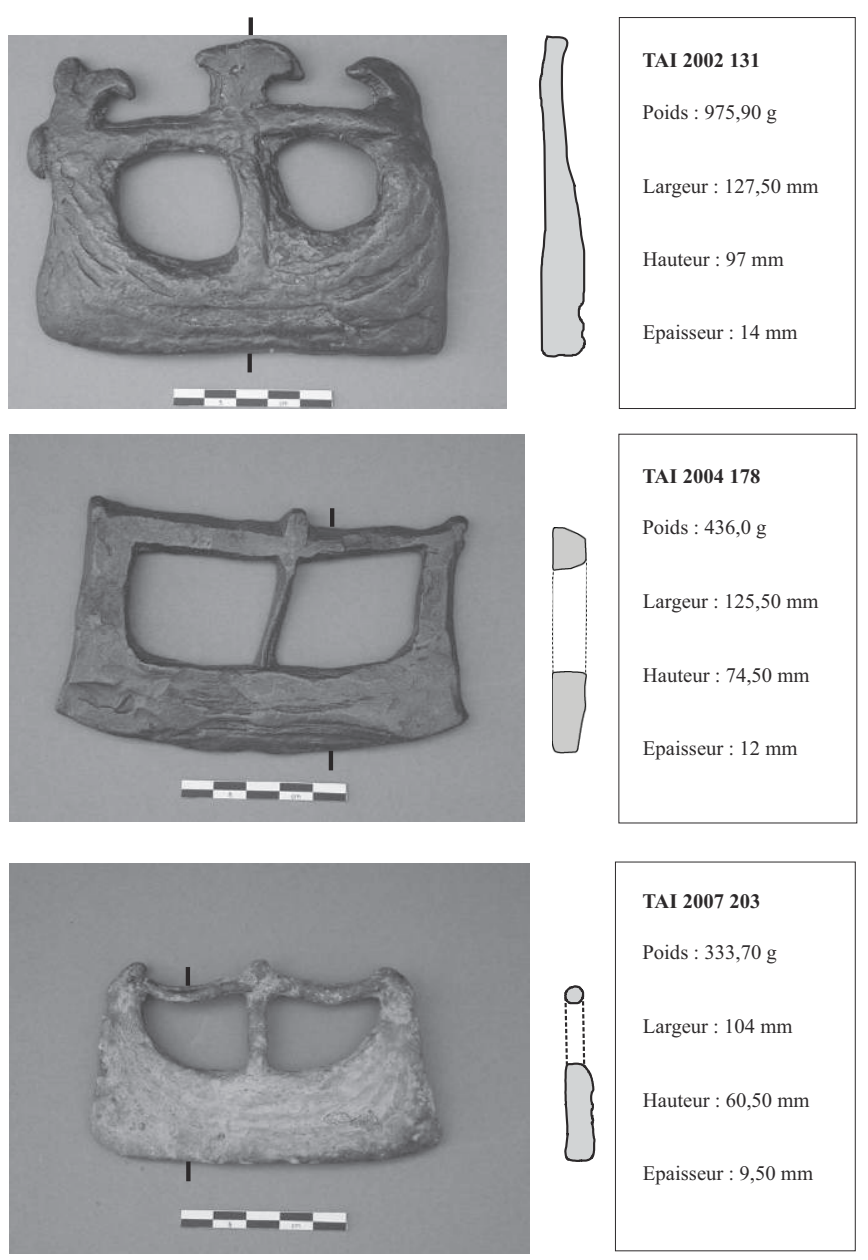
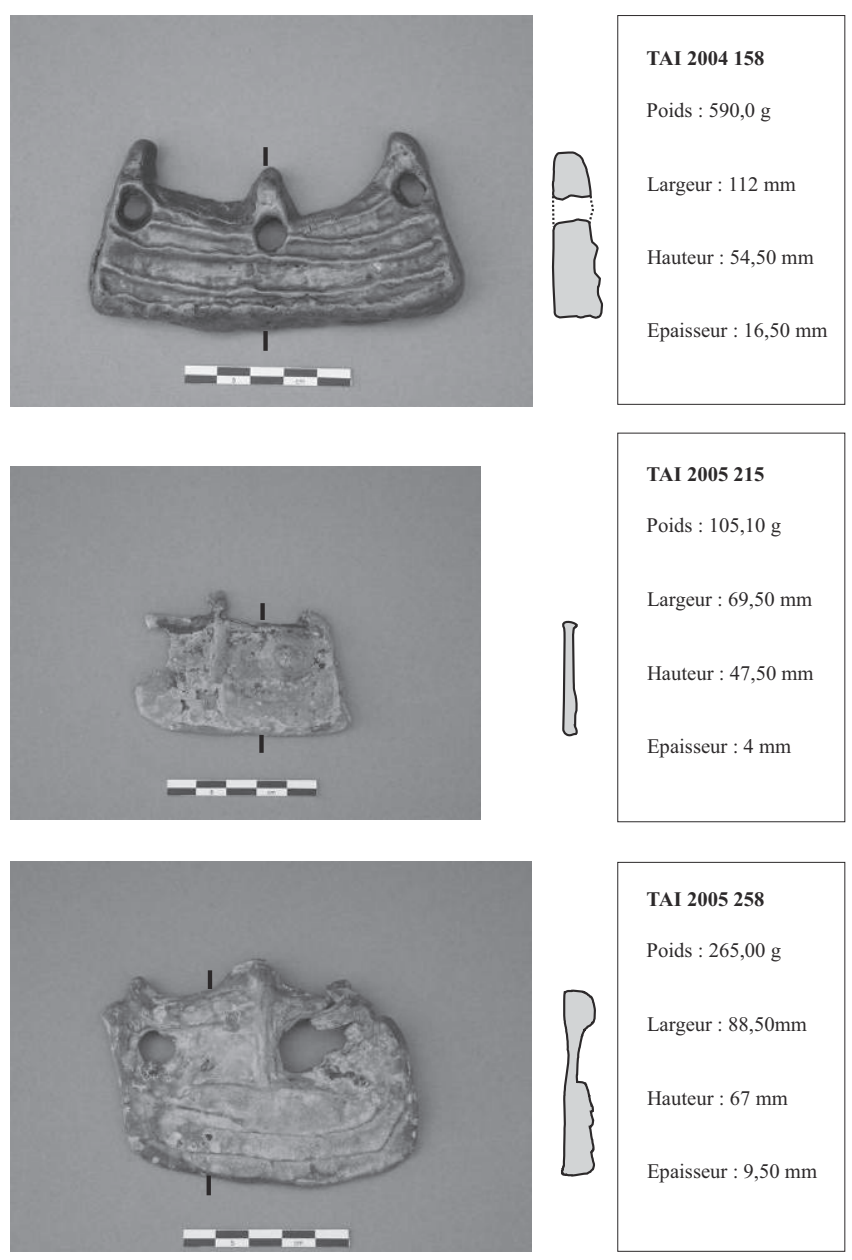

Figure 6 : Les plombs naviformes.

Figure 6: boat-form leads.

en œuvre semble plus reposer sur l'usage d'une fonte au sable que sur d'autres méthodes.

\section{Les déchets de fonte}

La découverte de coulures de plomb témoigne de la présence d'une activité métallurgique secondaire. Ces déchets de fonte ont pu se former à l'occasion de renversements du creuset en cours de manipulation, où, plus sûrement, être la somme des pertes dans le foyer. Le plomb s'accumule en fond de four et emprisonne des charbons de bois. Il peut également s'agir de ratés de coulée ou d'une vidange du creuset après usage. Mais quelque soit l'origine de la formation de ces coulures, elles témoignent bien de l'activité métallurgique qui s'est déroulée à proximité du fleuve. Tout comme l'atteste l'existence au sein des plombs naviformes d'un lingot en cours de refonte.
Si pour l'Antiquité, le plomb est un métal courant, la période médiévale semble connaitre une certaine pénurie qui a été caractérisée dans une vision macro-économique aussi bien par la géochimie (Hong et al., 1994) que par l'étude archivistique (Madeline, 2009). Pourtant, ces plombs du haut Moyen Âge trouvés dans le fleuve Charente ne paraissent pas s'inscrire dans cette économie de pénurie dont il est question à l'échelle de l'Europe médiévale. Les déchets retrouvés à Taillebourg représentent une masse de $454 \mathrm{~g}$. Ils témoignent du peu de cas qui était fait de ce matériau. Alors que le lieu de production n'est pas localisé, ces rebuts et résidus de fonte représentent déjà $3 \%$ en masse du corpus ce qui est loin d'être négligeable ${ }^{3}$. Ces pertes, qui apparais-

3. Il n'existe pas d'éléments de comparaison avec des ateliers de plombier et pour cause. Nous basons notre comparaison sur des ateliers travaillant le cuivre où la perte mise au jour lors des fouilles représente entre 10 et $20 \%$ en masse du corpus. 
sent élevées, posent bien la question de l'accès à ce matériau. Dans une vision micro-économique à laquelle semble correspondre les pratiques halieutiques sur les bords de la Charente, le plomb ne peut pas être considéré comme un matériau rare d'autant plus que le site se trouve à proximité d'une zone de production de métal neuf (Melle).

\section{Les pièces isolées}

\section{Plombs massifs}

Sous cette dénomination se trouvent regroupées deux pièces massives dont le trait commun est l'existence de deux trous de suspension. Dans le cas de l'exemplaire TAI04-43, le diamètre des ouvertures rentre dans la gamme de ce qui a été observé pour les plombs à trois anses.

\section{Barre}

Exemplaire unique d'une barre de plomb avec un percement des passants dans l'axe longitudinale de la pièce (fig. 4). Il s'agit d'une feuille épaisse qui a été repliée sur elle-même de manière à former un parallélépipède auquel est donnée une légère courbure afin de limiter l'usure de la corde engagée dans les passants.

\section{Plombs circulaires}

Il s'agit sûrement de la plus simple expression d'un poids de suspension. La forme est circulaire. Le passant est réalisé dans un cas lors de la coulée avec une réserve et, dans les deux autres, à froid par poinçonnage. L'emplacement de l'œil est variable (centre et périphérie) sans que l'on puisse en déduire une différence dans la fonction.

\section{Masses informes}

Les quatre objets qui constituent ce groupe se définissent soit par la mauvaise qualité de la coulée due à un manque de métal, soit au choix du support de coulée. Pour l'exemplaire TAI04-154, il semble que nous soyons dans le cadre d'un réemploi d'une pièce déjà formée.

\section{Divers objets}

Les prospections ont également conduit à la découverte d'un jeton de plomb avec des traces de pincement, d'un tube formé à l'aide d'une feuille roulée sur elle-même et d'une bille avec une perforation cylindrique de $4,25 \mathrm{~mm}$ de diamètre.

\section{RECHERCHE SUR L'ORIGINE DU MÉTAL}

Dans l'espace picto-charentais et pour la période qui nous intéresse, il existe une exploitation de galène argentifere. Il s'agit des mines de Melle en activité entre les $\mathrm{VI}^{\mathrm{e}}$ et $\mathrm{X}^{\mathrm{e}}$ siècles (Téreygeol, 2001). Si ce minerai a été exploité en premier lieu pour l'argent qu'il était susceptible de fournir, le plomb est un sous-produit majeur de cette activité. En effet, la production d'un kilogramme d'argent entraîne nécessairement la production de près d'une centaine de kilogramme de plomb. Le minerai titre en moyenne 1 à $3 \%$ d'argent. La production de plomb pourrait être nettement supérieure mais les pertes lors de la fusion réductrice puis lors de la revivification des litharges sont telles que l'on ne peut espérer recueillir en moyenne qu'un dixième du plomb disponible dans le minerai. Le plomb dans cet espace géographique, et pour la période considérée, n'est donc pas une denrée rare. Il est évident également que si les Scandinaves se sont intéressés à Melle, comme nous le trouvons relaté dans les annales de Saint-Bertin, ce n'était évidemment pas pour le plomb mais bien pour l'argent. Néanmoins, dans une perspective de recherche portant sur la diffusion des sous-produits de la métallurgie de l'argent de Melle, il est pertinent de poser la question de l'origine de ces plombs retrouvés au fond du fleuve Charente puisque nous avons déjà pu mettre en évidence une étonnante circulation du verre de scorie (Gratuze et al., 2003).

Il n'y a pas lieu de détailler ici la méthode d'analyse isotopique qui permet d'établir une filiation entre un minerai et un objet par l'intermédiaire du plomb. Précisons simplement que les analyses ont été conduites en collaboration avec messieurs P. Horn et $S$. Holzl de l'Institut Für Mineralogie, Petrologie und Geochemie de l'Université LudwigMaximilian à Munich. Le travail en laboratoire a été effectué par TIMS (Thermal Ionisation Mass Spectrometry). La marge d'erreur pour les rapports $\mathrm{Pb} 206 / 207$ et $\mathrm{Pb} 208 / 207$ est de $0,05 \%$, de $0,1 \%$ pour les rapports $\mathrm{Pb} 206 / 204$ et de 0,15\% pour le rapport $\mathrm{Pb} 207 / 204$.

Le corpus analysé porte sur neuf objets : sept plombs naviformes et deux masses de plomb, l'une portant trois yeux, l'autre un seul. Le choix s'entend eu égard à la forme spécifique de ces objets. Bien que tous aient été trouvés dans le même contexte, il semblait pertinent de s'intéresser à la famille des plombs dont la forme évoque un bateau stylisé assez caractéristique des représentations connues dans le monde scandinave (Mariotti et al., 2005 et 2006). Les résultats sont comparés au domaine isotopique du minerai de Melle qui a déjà été caractérisé à d'autres fins (Téreygeol, 2004).

Sur les 9 pièces étudiées, trois ont des rapports isotopiques très semblables : TAI04-C54, TAI04-158 et TAI0448 (fig. 7). Elles pourraient non seulement provenir d'un 


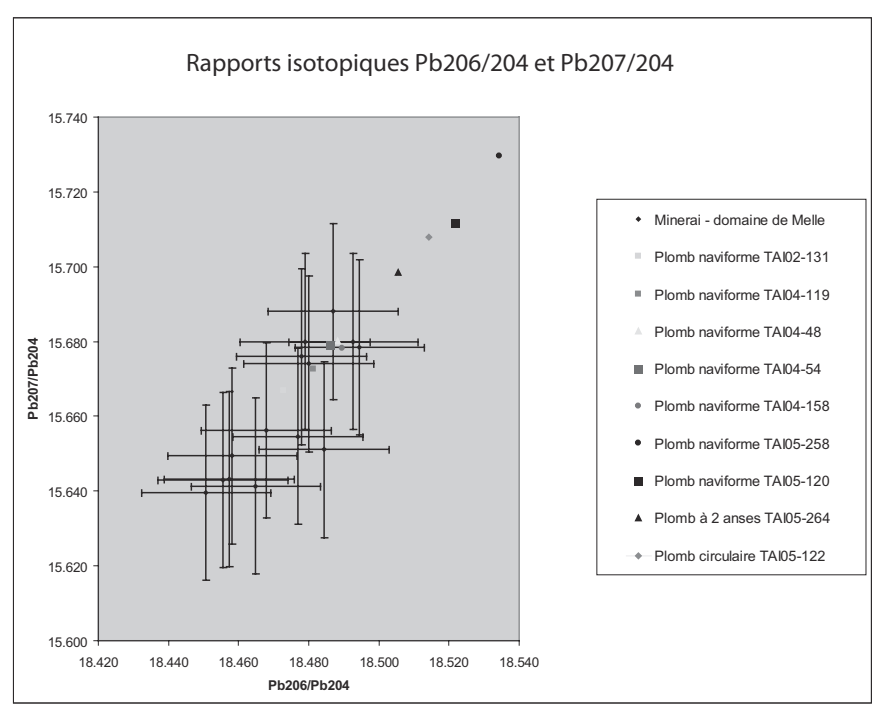

Figure 7 : (voir planche couleur) Tableaux des rapports isotopiques. Figure 7: (see colour plate) Isotopic ratio.

plomb issu des mines de Melle mais avoir été coulées à partir d'un même lot métallique. Quatre autres pièces (TAI04-119, TAI02-131, TAI05-264 et TAI05-122) appartiennent également au domaine du minerai de Melle. Leur dispersion au sein du domaine qualifie des apports en plomb non mellois réels mais restant très limités. Enfin deux pièces (TAI05120 et TAI05-258) sortent légèrement du domaine mellois. Néanmoins les rapports isotopiques de ces deux derniers plombs se placent sur l'axe moyen du domaine considéré. Une conclusion s'impose : en croisant les différentes informations archéologiques, chronologiques et isotopiques, il semble bien que le plomb de Melle ait servi à la fabrication de ces objets. Le plus étonnant reste la grande similitude des rapports isotopiques entre chaque plomb qui traduit le faible impact des plombs allogènes. De deux choses l'une, ou ces plombs ont été fabriqués dans un laps de temps relativement court à partir d'un stock de métal unique, ou l'importance de la production melloise fait que, sur un temps plus long, on observe toujours la même signature. La première hypothèse ne peut être ni étayée ni démontrée en l'état des connaissances sur le site de Taillebourg et des alentours. En revanche, la seconde apparait comme la plus simple d'autant plus qu'elle se trouve renforcée par les premières constatations effectuées sur le monnayage immobilisé de Melle (Téreygeol et al., 2005). Ce dernier, bien que produit principalement après l'abandon des mines s'apparente toujours au domaine isotopique du plomb mellois. Les facteurs conjugués d'une forte production durant 500 ans et d'une circulation monétaire limitée conduisent, près de 200 ans après la cessation de l'extraction, à toujours trouver la signature

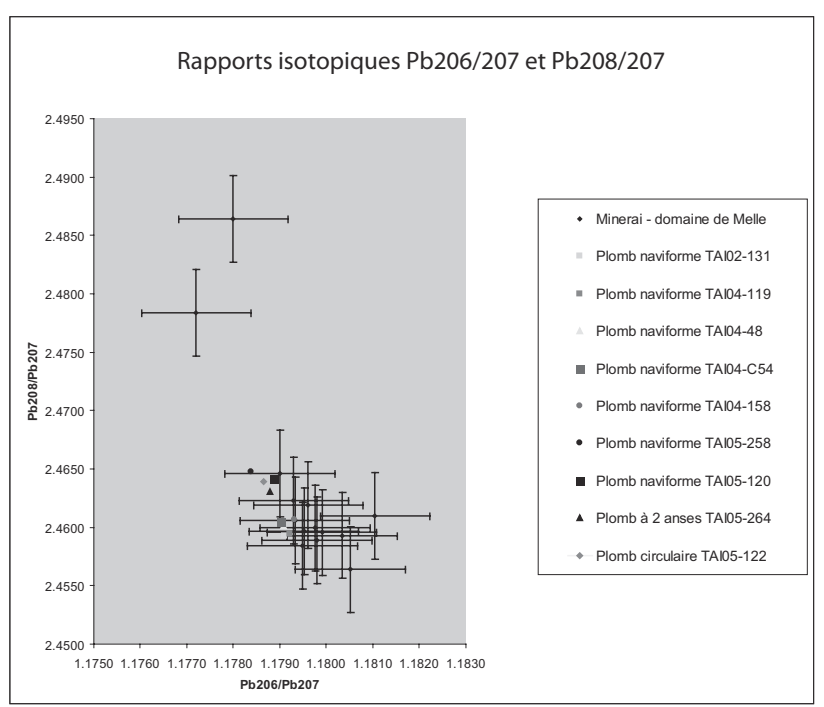

isotopique du plomb de Melle dans les monnaies poitevines par le simple jeu des refontes sans apport notable de métal allogène. Le cas de Taillebourg pourrait trouver la même origine d'autant plus que ce matériel apparaît alors même que les mines sont en pleine activité. Nous sommes dans le cas relativement simple, où sur une zone de production, le métal de primo extraction marque largement de sa signature les objets en plomb oblitérant assez facilement les apports extérieurs. Ceci nous permet de poser un premier jalon pour définir la zone d'influence de la mine de Melle en relation avec sa production de métal gris. Cette limite s'étend au moins vers le sud jusqu'à $60 \mathrm{~km}$ du gisement mellois.

\section{LA NATURE DU MÉTAL}

La question de la composition du métal ayant servi à la fabrication de ces plombs est abordée au travers d'une première étude faite par fluorescence $\mathrm{X}$ à l'aide de deux appareillages distincts : un générateur de rayons $\mathrm{X}$ à anode tournante Rigaku RU - 200BH disposant d'une anode au molybdène et un générateur portable Nilton Xlt. Si le choix de l'analyse par fluorescence $\mathrm{X}$ a l'avantage de la rapidité, les résultats obtenus sont nécessairement limités eu égard à la méthode d'analyse choisie (fig. 8). C'est d'ailleurs ces limitations qui ont nécessité le recours à deux instruments différents pour élargir quelque peu le champ des possibles.

Dans le cadre d'une analyse de plomb, plusieurs éléments peuvent être recherchés pour répondre à différentes interrogations : l'argent pour la qualité de l'affinage (Rehren et 
Tableau A

\begin{tabular}{|c|c|c|c|}
\hline $\mathbf{N}^{\circ}$ & Type de plomb & Cu en ppm & Zn en ppm \\
\hline Tai03160 & naviforme & 930 & 315 \\
\hline Tai03387 & plomb à 4 bras & 380 & 55 \\
\hline Tai03420 & plomb à 4 bras & 390 & nd \\
\hline Tai03359 & plomb à 4 bras & 440 & nd \\
\hline Tai03386 & plomb à 4 bras & 440 & 120 \\
\hline Tai03154 & plomb à 4 bras & 490 & nd \\
\hline Tai03422 & plomb à 4 bras & 640 & 80 \\
\hline Tai03182 & plomb à 4 bras & 670 & 160 \\
\hline Tai03302 & plomb à 4 bras & 700 & nd \\
\hline Tai03314 & plomb à 4 bras & 860 & 180 \\
\hline Tai03161 & plomb à 4 bras & 900 & 200 \\
\hline Tai03155 & plomb à 4 bras & 1100 & 160 \\
\hline Tai03421 & plomb à 4 bras & 1100 & nd \\
\hline Tai03434 & plomb à 2 anses & 1200 & 100 \\
\hline Tai03454 & plomb à 2 anses & 730 & nd \\
\hline Tai03455 & plomb à 2 anses & 700 & nd \\
\hline Tai03456 & Masse informe & 550 & 100 \\
\hline Tai04334 & Coulure & 990 & nd \\
\hline Tai03152 & divers & 830 & nd \\
\hline Tai03199 & divers & 600 & nd \\
\hline
\end{tabular}

Tableau B

Figure 8 : Liste des résultats analytiques (tab. A : résultats acquis par un générateur de rayons $\mathrm{X}$ à anode tournante Rigaku RU - 200BH disposant d'une anode au molybdène; tab. $\mathrm{B}$ : résultat acquis par un générateur portable Nilton Xlt).

Figure 8: Analytical results (tab. A: results acquired with $X$-ray generator Rigaku $R U-$ 200BH; tab. B: results acquired with X-ray generator Nilton $X l t$ ).

\begin{tabular}{|l|c|c|c|c|}
\hline \multicolumn{1}{|c|}{$\mathbf{N}^{\mathbf{0}}$} & Type de plomb & Cu en ppm & Zn en ppm & Sn en ppm \\
\hline Tai05214 & plomb circulaire & nd & nd & nd \\
\hline Tai05292 & plomb à 2 anses & nd & nd & nd \\
\hline Tai05264 & plomb à 2 anses & nd & nd & 12600 \\
\hline Tai05258 & naviforme & nd & nd & 5100 \\
\hline Tai05120 & naviforme & nd & nd & nd \\
\hline Tai05215 & naviforme & nd & nd & nd \\
\hline Tai05133 & plomb à 4 bras & nd & nd & nd \\
\hline Tai05136 & plomb à 4 bras & nd & nd & nd \\
\hline Tai05114 & plomb à 4 bras & nd & nd & 19400 \\
\hline Tai05159 & plomb à 4 bras & nd & nd & 12300 \\
\hline Tai05110 & plomb à 4 bras & nd & nd & nd \\
\hline Tai05134 & plomb à 4 bras & nd & nd & nd \\
\hline Tai05180 & plomb à 4 bras & nd & nd & 24700 \\
\hline
\end{tabular}

Prange, 1998), le cuivre, l'étain et le zinc pour définir de possibles refontes et ajouts, le bismuth dont les fortes concentrations sont réservées au plomb moderne (Cuzange et Texier, 2000). Dans le cas spécifique des plombs de Charente et au vu des résultats des analyses isotopiques, les données peuvent être croisées avec les teneurs observées pour le plomb de Melle qui contient en moyenne 0.1 à $0.3 \%$ mass d'argent, 200 à 400 ppm de cuivre, un maximum de 400 ppm de zinc, et dont l'étain est quasi absent (Téreygeol, 2001). Les limites des appareils associées à la nature des matrices analysées font que nous ne sommes en mesure d'apporter des résultats que pour le cuivre et le zinc pour le générateur de rayons $\mathrm{X}$ à anode tournante auxquels s'ajoute l'étain pour le générateur portable (L’Héritier, 2009) ${ }^{4}$. Malgré cette limitation à seulement trois éléments, il ressort des informations quant à la nature de ces plombs.

Les travaux récents sur la composition des plombs ont montré une tendance à un enrichissement en cuivre des plombs depuis l'antiquité jusqu'à la période moderne

4. Les éléments que nous pouvons doser à l'aide de nos appareillages sont le cuivre (limite de détection : 50 ppm pour le générateur Rigaku et $1000 \mathrm{ppm}$ pour le générateur Nilton), l'étain (limite de détection : non définie pour le générateur Rigaku et $4000 \mathrm{ppm}$ pour le générateur Nilton), l'antimoine (limite de détection : $200 \mathrm{ppm}$ pour le générateur Rigaku et supérieure à $1300 \mathrm{ppm}$ pour le générateur Nilton), le zinc (limite de détection : $50 \mathrm{ppm}$ pour le générateur Rigaku et supérieure à 1300 ppm pour le générateur Nilton). 
(L'héritier, sous presse; Téreygeol, 2009). En utilisant ce classement, nous trouvons parmi les vingt objets analysés via le générateur de rayons $\mathrm{X}$ à anode tournante deux objets avec un taux de cuivre compatible avec ceux observés pour l'Antiquité (entre 200 et 400 ppm) et dix avec un taux compris entre 400 et 800 ppm qui correspondrait à celui observé pour les plombs antérieurs au $\mathrm{x}^{\mathrm{e}}$ siècle. Deux plombs ont un taux légèrement supérieur au seuil limite de 800 ppm et trois se démarquent nettement avec une concentration en cuivre supérieure à 1000 ppm (seuil caractérisant des plombs plus tardifs). Dans ce corpus limité, la concentration en cuivre placerait ces plombs au haut Moyen Âge. La prudence s'impose à ce stade car les dosages supérieurs à 600 ppm montrent également que nous sommes au-delà du domaine de solubilité du cuivre dans le plomb et des effets de pépites ne sont pas à exclure. Ainsi nous préférerons parler d'une tendance qui reste à caractériser plus finement par l'élargissement du corpus.

Les taux de zinc qui ont été observés sont proches de la limite de détection. Pour plus de la moitié des cas, nous ne pouvons certifier que ce métal soit totalement absent du plomb. En revanche, les taux que nous avons pu mesurer s'inscrivent tous dans la moyenne attendue au regard du minerai mellois. Il n'est donc pas possible à l'aide de ce seul élément de mieux caractériser la nature ni la provenance du métal sauf à dire qu'il ne semble pas provenir d'une activité de refonte très prononcée et reste compatible avec une origine melloise.

Un deuxième corpus a été dosé à l'aide du second générateur de rayons $\mathrm{X}$. Le même travail n'a pu être conduit, la qualité de détection pour le cuivre et le zinc étant nettement moins bonne. En revanche, nous avons accès au dosage de l'étain dont la présence marque les phénomènes de refonte si caractéristiques, semble-t-il, des plombs du Moyen Âge. Parmi les treize objets analysés, cinq plombs affichent des taux d'étain sans commune mesure avec le minerai de Melle. Il est alors possible d'affirmer que ces plombes ne proviennent pas directement des fonderies melloises mais bien d'une activité de recyclage.

Notons enfin que sur trois pièces, il y a eu à la fois un dosage isotopique du plomb et une analyse par fluorescence X (Tai 05-264, 120 et 258). Il est remarquable que les deux plombs qui s'écartent du domaine isotopique de Melle (Tai 05-264 et 258) soient également ceux présentant des taux élevés d'étain. Ici encore, cette corrélation est de bon augure mais demanderait à être renforcée par la multiplication des exemples.

\section{Conclusion}

Au terme de cette première étude, plusieurs conclusions peuvent être avancées. Il apparaît que ces pièces ont pu facilement être fabriquées sur place. Il s'agit bien sûr d'une métallurgie secondaire et non extractive. Le métal utilisé est homogène, provenant en majorité d'une seule et même source. La filiation entre ce métal et le minerai de Melle est posée. Il pourra être intéressant d'observer à partir de quelle distance, le signal mellois se perd mais à l'heure actuelle, le matériel pour une telle étude fait défaut.

Sur le plan de la mise en forme, une partie du mobilier est directement produite par moulage mono-valve. L'aspect des surfaces coulées en contact avec le moule pourrait indiquer à la fois l'usage d'un sable de fonderie mais également de bois et, dans quelques cas, d'une coulée à même le sol. Certaines pièces sont plus élaborées et nécessitent une coulée suivie d'une mise en forme par martelage mais au final les techniques mises en œuvre restent simples et pourraient très bien prendre place dans un espace qui ne soit pas spécialement dévolu au travail du plomb, ni même au travail du métal en général.

La poursuite des analyses est nécessaire afin de mieux saisir la composition globale de ces plombs. Si l'analyse à l'aide d'un générateur de rayons $\mathrm{X}$ possède d'indéniables avantages, elle est loin de pouvoir répondre à toutes nos interrogations.

Sur ce corpus particulier, une mise en situation expérimentale dans le lit du fleuve (accrochage, suspension, immersion) pourrait renseigner sur certains stigmates et sur la durée de vie de tels objets. Ces expérimentations in situ devront se comprendre sur un temps long et prendre appuis à la fois sur nos hypothèses mais aussi sur des exemples ethnographiques.

\section{Bibliographie}

Arles, A. et Téreygeol, F., 2009. La fonte des alliages dans les ateliers monétaires médiévaux : l'exemple de MontreuilBonnin (Vienne), Numismatique et Archéologie en PoitouCharentes, coll. « Recherches et Travaux de la Société d'Études Numismatiques et Archéologiques " n 2, p. 129-148.

Baron, S. et Cochet, A., 2003. Étude d'un lot de lingots de plomb antiques des Saintes-Maries-de-la-Mer : ségrégations et analyses chimiques élémentaires, Revue d'archéométrie, $\mathrm{n}^{\circ} 27$, p. 147-163.

Cochet, A., 2000. Le plomb en Gaule romaine, coll. «Instrumentum », 13, ed. Monique Mergoil. 
Cuzange, L. et Texier, A., 2000. Caractérisation des plombs anciens de vitraux, Corpus Vitrearum Newsletter, $\mathrm{n}^{\circ} 47$, p. 43-51.

Debord, A., 1984. La société laïque dans les pays de la Charente, $X^{e}$-XII siècles, Paris, Picard.

Dumont, A. (dir.), 2007. Approche archéologique, environnementale et historique du fleuve Charente. Etude interdisciplinaire et diachronique du lit mineur et des berges entre Saintes et Taillebourg - Port d'Envaux. Rapport d'activité 2006 déposé au Service Régional de l'Archéologie de Poitou-Charentes, Poitiers.

Gratuze, B., Téreygeol, Fl., Lancelot, J. et Foy, D., 2003. Is there a relationship between some medieval lead-glass and the glassy slag produced by lead-silver mines, Archaeometallurgy in Europe, international conference, vol. 2, Milan, p. 513-519.

Gruat, Ph. (dir.), 2003. Du silex au Métal, catalogue d'exposition, Musée Archéologique de Montrozier, Guide archéologique $\mathrm{n}^{\circ} 9$.

Hong, S., Candelone, J.-P., Patterson, C. et Boutron, C., 1994. Greenland Ice Evidence of Hemispheric lead pollution two millennia ago by greek and roman civilizations, Science, Vol. 265, p. 1841-1843.

L'Héritier, M., 2009. La problématique de l'analyse du plomb, Monumental, p. 104-106.

L'Héritier, M., sous presse. Le fer et le plomb dans l'architecture médiévale : composition, origines technique et géographique. Question à l'Archéométrie, Cahiers de Recherches Médiévales.

Lautier, C. et SAndron, D. (dir.), 2008. Antoine de Pise : l'art du vitrail vers 1400 , Paris, CTHS.

Madeline, F., 2009. Le don de plomb dans le patronage monastique d'Henri II Plantagenêt : usages et conditions de la production du plomb anglais dans la seconde moitié du XII ${ }^{\mathrm{e}}$ siècle, Archéologie Médiévale, t. 39, p. 31-52.

Mariotti, J.-F., Dumont, A., Mathé, V., Camus, A., Lévêque, F., Nissen-Jaubert, A., Hulot, O., Greck, S. et Szepertyski, B., 2005. Prospection du lit mineur et des berges sur le site médiéval de Taillebourg - Port d'Envaux (Charente-Maritime) : un secteur d'activité lié à la Charente, Aquitania, XXI, p. 299336.
Mariotti, J.-F., Dumont, A. et Nissen-Jaubert, A., 2006. Une zone portuaire médiévale à Taillebourg - Port d'Envaux (fleuve Charente, France) in Hafner, A., Niffeler, U. et Ruoff, U. (dir.), L'apport de l'archéologie subaquatique, Actes du $2^{\mathrm{e}}$ Congrès International d'Archéologie Subaquatique, Zurich, octobre 2004, (Antiqua 40), p. 220-229.

Rehren, T. et Prange M., 1998. Lead metal and patina: a comparison, Der Anscnhitt, Beiheft 8, p. 183-196.

Téreygeol, Fl., 2001. Les mines d'argent carolingiennes de Melle, thèse de $3^{\mathrm{e}}$ cycle, Université de Paris 1 Panthéon-Sorbonne, 3 vol.

Téreygeol, Fl., 2007. Production and circulation of silver and secondary products (lead and glass) from frankish royal silver mines at Melle $\left(7^{\text {th }}-10^{\text {th }}\right.$ century), dans J. Henning (ed.) Postroman towns and trade in Europe, byzantium and the near-East, vol. 1, p. 123-134.

Téreygeol, Fl. (dir.), 2009. Le plomb dans les monuments historiques au Moyen Âge : étude de la filiation et de la diffusion d'un nouveau procédé technique, le saïger prozess, Rapport de synthèse, Programme National de Recherche sur la Connaissance et la Conservation des Matériaux du Patrimoine Culturel.

Téreygeol, Fl., Hoelzl, S. et Horn, P., 2005. Le monnayage de Melle au haut Moyen Âge : état de la recherche, Bulletin de l'AAPC, $\mathrm{n}^{\circ} 34$, p. 49-56.

Téreygeol, Fl. et Foy, E., 2005. Les plombs de Taillebourg - Port d'Envaux (17), Rapport d'étude archéométrique, Rapport déposé au SRA de Poitou-Charentes à Poitiers et à l'Institut de recherche sur les archéo-matériaux, Orléans.

Téreygeol, Fl. et Foy, E., 2006. Les plombs de Taillebourg - Port d'Envaux (17). Rapport d'étude archéométrique, Rapport intégré au rapport de PCR (Dumont [dir.], 2007), déposé au SRA de Poitou-Charentes à Poitiers.

Wallace, P. F., 1998. Line fishing in Viking Dublin: a contemporary explanation for archaeological evidence, in Dublin beyond the pale, edited by Conleth Manning, Wordwell in association with Rathmichael Historical Society, p. 3-18. 
